\title{
Primera cita de nidificación de pico mediano (Dendrocoptes medius, Linn. 1758) en la sierra de Elgea-Urkilla (Álava).
}

\section{First record of a nesting pair of middle spotted woodpeckers (Dendrocoptes medius, Linn. 1758) on the Elgea-Urkilla mountain range (Alava).}

Maite Martinez-Madrid ${ }^{1,2}$, Javier Sesma ${ }^{3}$, José M. Fernández-García ${ }^{2,4}$

\section{*}

\section{Resumen}

Se reporta la primera cita de nidificación conocida de pico mediano (Dendrocoptes medius, Linn. 1758) en la sierra de Elgea-Urkilla (Álava). Esta observación tiene trascendencia para determinar mejor la distribución, el patrón de ocupación y los factores que condicionan ambas, en el conjunto de la población del País Vasco.

Palabras clave: Picidae, reproducción, País Vasco, especie vulnerable.

\section{Abstract}

In this article we report the first known breeding record of the middle-spotted woodpecker (Dendrocoptes medius, Linn. 1758) on the Elgea-Urkilla mountain range (Alava). This observation is important as it allows us to determine the distribution and occupation patterns

\footnotetext{
1 Universidad del País Vasco UPV/EHU

Departamento de Genética, Antropología Física y Fisiología Animal.

Apdo 644, 48080. Bilbao

*Correspondencia: maite.martinez@ehu.eus

2 Instituto Alavés de la Naturaleza IAN

Pedro de Asúa, 2. 01008 Vitoria-Gasteiz

3 Diputación Foral de Álava

Departamento de Medio Ambiente y Urbanismo. Servicio de Patrimonio Natural.

Plaza de la Provincia, s/n. 01001 Vitoria-Gasteiz
}

4 Fundación Hazi

01192 Arkaute 
of this species as well as the factors that influence both of these parameters in the entire population of the Basque Country.

Key words: Picidae, reproduction, Basque Country, vulnerable species.

\section{Laburpena}

Okil ertainaren (Dendrocoptes medius, Linn. 1758) lehen habiaketa ezagunaren berri eman da Elgea-Urkilla mendilerroan (Araban). Behaketa hau garrantzitsua da hobeto zehazteko EAEko biztanleriaren banaketa, okupazio eredua eta biak baldintzatzen dituzten faktoreak.

Gako hitzak: Picidae, ugalketa, Euskadi, espezie ahula.

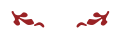

El pico mediano (Dendrocoptes medius, Linn. 1758) es un pájaro carpintero netamente forestal, que aparece principalmente ligado a masas arbóreas maduras de distintas especies caducifolias o marcescentes del género Quercus (Winkler et al., 2014). El pico mediano se distribuye fundamentalmente por la franja climática templada del Paleártico occidental, con poblaciones continuas en Europa central y oriental, y fragmentadas en las penínsulas mediterráneas (Pasinelli, 2003; Gorman, 2004).

En el País Vasco, la presencia del pico mediano fue mencionada por primera vez en 1994 (Arambarri y Rodríguez, 1996) y la especie ocupa fundamentalmente bosques de roble rebollo o marojo (Quercus pyrenaica) (Fernandez-Garcia, 2016). Las poblaciones conocidas hasta la fecha se encuentran en el sureste de Álava -incluyendo el Parque Natural de Izki-, los Montes de Vitoria y la sierra de Entzia (Onrubia et al., 2003; Fernandez-Garcia, 2016). Aunque la población española en su conjunto se considera "Casi amenazada" según criterios UICN (Onrubia et al., 2004), desde el punto de vista legal en el País Vasco el pico mediano fue catalogado como "Vulnerable" (Orden de 10 de enero de 2011, de la Consejera de Medio Ambiente, Planificacion Territorial, Agricultura y Pesca del Gobierno Vasco).

La sierra de Elgea-Urkilla, al norte de Álava, alberga un extenso (2.380 ha) robledal de $Q$. pyrenaica. En el curso de prospecciones con metodología específica (estaciones de escucha con reclamo) en la primavera de 1994, el pico mediano fue detectado en 3 cuadrículas de $1 \mathrm{~km}^{2}$ de un total de 13 muestreadas (Arambarri y Rodriguez, 1994). Las cuadrículas positivas se situaban en el sector más oriental de la sierra (UTM de

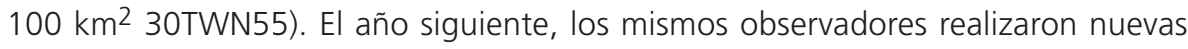
prospecciones en las mismas masas forestales y con el mismo método, pero la especie ya no fue localizada (Lanius et al., 1995; Arambarri y Rodríguez, 1996). Posteriormente se han efectuado en Elgea-Urkilla inventarios ornitológicos generalistas sobre unidades 
territoriales de 100 km², en 1998-2001 (Onrubia et al., 2003), 2002-2005 (Gainzarain, 2006) y 2014-2018 (SEO/BirdLife, no publ.), sin obtener registros de la especie. En marzo-abril de 2017-2018 se realizaron muestreos sistemáticos específicos (estaciones de escucha con reclamo) sobre cuadrículas de 1 km² (8 en 2017, 20 en 2018), de nuevo sin resultados positivos (Fernandez-Garcia, 2019). Tampoco hay constancia de observaciones oportunistas grabadas en los repositorios de citas avifaunísticas que cubren el País Vasco (www.ornitho.eus y www.eBird.org/spain). En resumen, a pesar de haberse efectuado esfuerzos de búsqueda, planificados, específicos y a distintos niveles de resolución, la especie no parece haber sido detectada en el área de estudio desde 1994 (Unanue et al., 2014; Fernández-García, 2016).

El 16 de abril de 2020, una de las autoras (M.M.M.) observó un ejemplar de pico mediano con comportamiento territorial, emitiendo reclamo de manera insistente en una mancha boscosa de la sierra de Elgea-Urkilla (UTM 30TWN45), lo que se repitió durante varios días. A partir de ese momento se realizó un seguimiento exhaustivo del territorio, comprobándose la fijación de los ejemplares al mismo.

El 23 de abril se detectó el inicio de la construcción del nido en el tronco principal de un pie de Q. pyrenaica parasitado por Phellinus robustus, perímetro de $75 \mathrm{~cm}$ (medido a 1 metro del suelo), altura total $12 \mathrm{~m}$ (aprox.) y diámetro de copa 4,20 m. El agujero del nido se orientaba al sur, a una altura de 3,16 m, diámetro de entrada 4,5 cm, profundidad horizontal $10,5 \mathrm{~cm}$ y profundidad vertical $33,5 \mathrm{~cm}$. La construcción del nido se prolongó durante 13 días, en los que se observó de manera puntual a ambos miembros de la pareja juntos, con comportamiento discreto (Fig. 1A). Durante este periodo uno de los ejemplares pasó gran parte del día picando y sacando virutas con el pico. Posteriormente no se apreció aporte de material exógeno al nido. Una vez finalizada la construcción, uno de los ejemplares se mantuvo casi de manera permanente en el nido, ausentándose solo durante intervalos de tiempo breves para alimentarse. Entre el 15 y el 23 de mayo se observó que los progenitores realizaban relevos de turnos en la incubación, periodo que se estimó en 12 días aproximadamente.

Durante 9 días (26 mayo-3 junio) se monitorizaron las cebas a los pollos, realizando observaciones en periodos de 1 hora $(n=8)$, encontrándose que el número de cebas por hora varió entre 1 y 8 (media: 4,38 cebas/hora; desviación estándar: 2,56). Este valor es menor que el reportado por Arambarri y Rodríguez (1997) en dos nidos de pico mediano monitorizados en Izki (4-15 cebas/hora, promedio 9 cebas/hora). Los aportes estaban compuestos exclusivamente por invertebrados, sin que se observaran cebas con bayas de hiedra (Hedera helix) u otro material vegetal, que sí son habituales en Izki (Arambarri y Rodríguez, 1997; POCTEFA Habios, no publ.) y en algunas poblaciones centroeuropeas (Spühler et al., 2015).

Los adultos alimentaron a los pollos hasta el día 4 de junio. En esta fecha se dejaron de observar cebas. Dos días después, se inspeccionó el interior del nido con cámara endoscópica, lo que permitió detectar dos pollos muertos, aún poco emplumados, de 


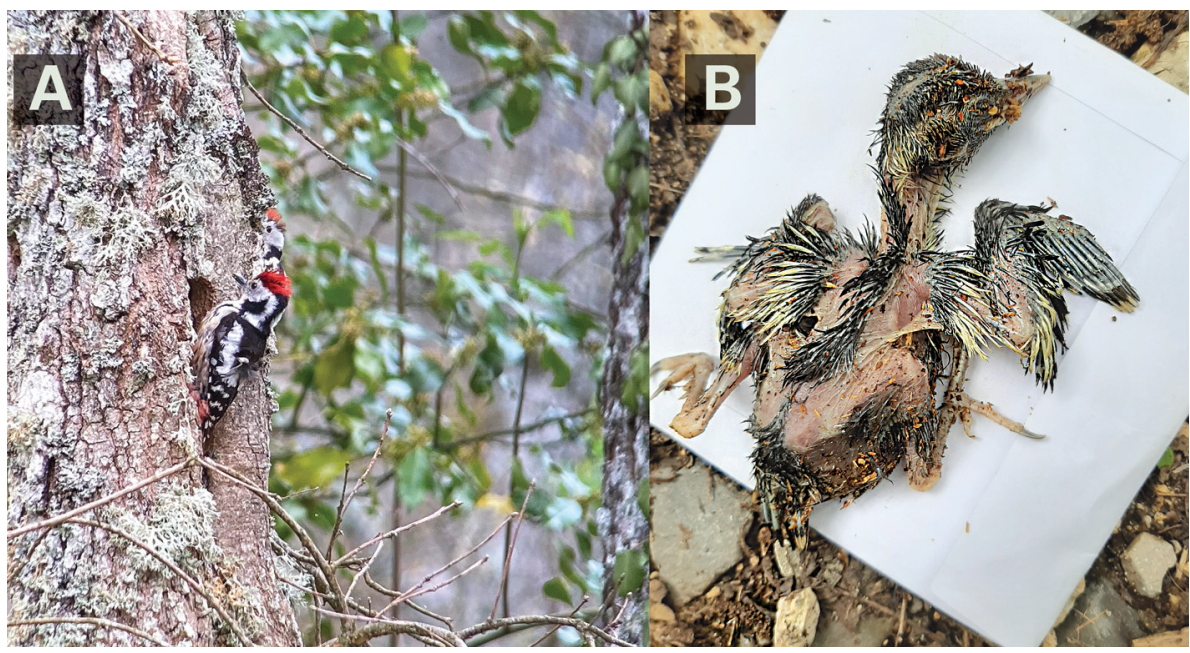

Fig. 1.- (A) Pareja nidificante de pico mediano en la Sierra de Elgea-Urkilla (Álava) durante la construcción del nido (Autor foto: Iñaki Arrate). (B) Pollo de pico mediano muerto, extraído del nido (Autor foto: José M. Fernández-García).

Fig. 1.- (A) Middle-spotted woodpecker nesting pair on the Elgea-Urkilla mountain range (Alava) during nest excavation (name of photographer: Iñaki Arrate). (B) Dead middle-spotted woodpecker chick, removed from the nest (name of photographer: José M. Fernández-García).

9-11 días de edad aproximadamente (Fig. 1B). No se apreciaron indicios de depredación, ni de usurpación del agujero por otra especie de ave o mamífero. El día 4 de junio se alcanzaron temperaturas mínimas de $9^{\circ} \mathrm{C}$ y precipitación de $12 \mathrm{l} / \mathrm{m}^{2}$, siguiendo a una semana inusualmente fría y lluviosa (Euskalmet, Agencia Vasca de Meteorología; https://www.euskalmet.euskadi.eus). No obstante, la causa concreta de la muerte de los pollos no pudo ser determinada.

El tamaño de pollada observada en el nido de la sierra de Elgea-Urkilla (2 pollos) fue menor al promedio descrito en la Cordillera Cantábrica $(3,1$ pollos volantones por nido, rango $1-5, \mathrm{n}=40$, Robles et al., 2008) y en Izki (2,2 pollos/nido, rango $1-3, \mathrm{n}=5$, Fernández-García, 2016; 3,1 pollos/nido, rango 1-5, n=26, POCTEFA Habios, no publ.).

Durante el mes de julio se observó varias veces un ejemplar de pico mediano a $110 \mathrm{~m}$ del árbol nido, lo que hace pensar que los adultos no abandonaron el territorio de cría, al menos de forma inmediata.

La fecha prevista de primer vuelo de los pollos hubiera sido más tardía que el promedio descrito para la población de Izki, que se sitúa en torno al 9 de junio (en 2017-2018: POCTEFA Habios, no publ.; en 2012: Ciudad y Robles, 2013; en 1995-1997: Arambarri y Rodríguez, 1997), y más parecida a la encontrada en la cordillera Cantábrica por Robles et al. (2008; $21 \pm 1$ de junio, rango 8 de junio-13 de julio). 
Entre el 28 de abril y el 9 de mayo se realizaron muestreos intensos, mediante estaciones de escucha con reclamo, en el parche forestal concreto donde se había detectado este evento de reproducción, un robledal maduro de Q. pyrenaica de 688 ha. Se efectuaron 26 estaciones distribuidas homogéneamente en 7 cuadrículas UTM de $1 \mathrm{~km}^{2}$ próximas a la del territorio conocido. Se obtuvieron tres contactos con picos medianos, suficientemente alejados del nido conocido (685 m, 770 m y 2.715 $\mathrm{m}$ ) como para pensar que se trataba de ejemplares diferentes, por lo que no se descarta que en la primavera de 2020 haya habido otros territorios ocupados en este mismo parche. No obstante, estas prospecciones se realizaron ya fuera del periodo de máxima detectabilidad de la especie, por lo que serán necesarios futuros esfuerzos más sistemáticos.

La presencia de una población reproductora de pico mediano en la sierra de ElgeaUrkilla que fue sugerida en 1994 (Arambarri y Rodríguez, 1996), no ha podido ser confirmada posteriormente. Tratándose de una especie que no resulta particularmente difícil de detectar durante el periodo de máxima actividad (precría y reproducción), podría descartarse que una población estable y densa hubiera simplemente pasado desapercibida. Por otro lado, desde el punto de vista del hábitat, la masa de robledal de Elgea-Urkilla satisface los requisitos estructurales y espaciales que selecciona la especie (p. ej. en Izki: Ciudad y Robles, 2013).

Un escenario verosímil estaría asociado a dinámicas irregulares de ocupación-colonización-extinción de parches de hábitat en Elgea-Urkilla por parte de ejemplares de pico mediano. Las dificultades de colonización y establecimiento podrían deberse a la ausencia de conespecíficos -que proporcionan claves de atracción social para los ejemplares dispersantes- y/o a la falta de conectividad estructural -i. e. continuidad forestal- respecto a poblaciones fuente, en Izki o Montes de Vitoria-Entzia. Este tipo de factores han sido señalados a la hora de explicar patrones de ocupación irregular de parches concretos en poblaciones de pico mediano en León (Robles y Ciudad, 2012). La determinación de la situación del pico mediano en Elgea-Urkilla mejoraría el conocimiento del estado de conservación y de las perspectivas de la población de Álava en su conjunto.

\section{Agradecimientos}

Queremos agradecer a Alfonso López de Armentia por la identificación del hongo que parasita al árbol nido, así como a Iñaki Arrate, Esther Bernedo y Alberto Tirados por su colaboración en el trabajo de campo. El proyecto POCTEFA Habios (www.habios.eu) ha generado mucha información sobre el pico mediano en el País Vasco. 


\section{Bibliography}

Arambarri, R., Rodriguez, A., 1994. El pico mediano (Dendrocopos medius) en los montes de Izki. Informe inedito para Diputacion Foral de Alava.

Arambarri, R., Rodríguez, A., 1996. Distribución y estima poblacional del pico mediano (Dendrocopos medius) en Álava. Ardeola 43, 221-223.

Arambarri, R., Rodriguez, A., 1997. Selección del hábitat de nidificación y análisis de espectro alimenticio del pico mediano (Dendrocopos medius) en el marojal de Izki. Hontza Natur Elkartea. Informe inédito para Gobierno Vasco.

Ciudad, C., Robles, H., 2013. Inventario y caracterización ecológica de la población de pico mediano en la ZEPA de Izki (Álava). Informe inédito para LIFE+ Pro-Izki.

Fernandez-Garcia, J.M., 2016. The Middle-spotted Woodpecker Leiopicus medius in the Basque Country, Northern Spain: review of current ecological status. Ornis Hung. 24, 4254.

Fernandez-Garcia, J.M., 2019. Resultados del muestreo participativo de pico mediano en Álava y Gipuzkoa (2017-2018). Informe inédito para POCTEFA Habios.

Gainzarain, J.A., 2006. Atlas de las aves invernantes en Alava (2002-2005). Diputacion Foral de Alava, Vitoria.

Gorman, G., 2004. Woodpeckers of Europe. A Study of the European Picidae. Bruce Coleman, Middlesex.

Lanius, Itsas Enara, Hontza, 1995. Los picidos (Picidae) en la CAPV. Informe inedito para Gobierno Vasco.

Onrubia, A., Robles, H., Salas, M., Gonzalez-Quiros, P., Olea, P., 2003. Pico mediano Dendrocopos medius. En: Marti, R., Del Moral, J.C. (Eds.), Atlas de las aves reproductoras de España, 358-359. Ministerio de Medio Ambiente, Madrid.

Onrubia, A., Robles, H., Salas, M., González-Quirós, P. Pérez, P., 2004. Pico mediano. En: Madroño, A., González, C. Atienza, J.C. (Eds.), Libro rojo de las aves de España, 304-307. Ministerio de Medio Ambiente, Madrid.

Pasinelli, G., 2003. Dendrocopos medius Middle Spotted Woodpecker. BWP Update 5, 4999.

Robles, H., Ciudad, C., Vera, R., Olea, P.P., Matthysen, E., 2008. Demographic responses of middle spotted woodpeckers (Dendrocopos medius) to habitat fragmentation. Auk 125, 131-139.

Robles, H., Ciudad, C., 2012. Influence of habitat quality, population size, patch size, and connectivity on patch-occupancy dynamics of the Middle Spotted Woodpecker. Cons. Biol. 26, 284-293.

Spühler, L., Krüsi, B.O., Pasinelli, G., 2015. Do Oaks Quercus spp., dead wood and fruiting Common Ivy Hedera helix affect habitat selection of the Middle Spotted Woodpecker Dendrocopos medius? Bird Study 62, 115-119. 
Unanue, A., Salvador, M., De la Hera, I., Auzmendi, G., 2014. Current status of the Middle-spotted Woodpecker Dendrocopos medius in Araba (Basque Country). En: FernándezGarcía, J. M., Robles, H., Pasinelli, G., Rubines, J. (Eds.), $7^{\text {th }}$ International Woodpeckers Conference. Book of Abstracts, 53. Vitoria.

Winkler, H., Christie, D.A., Kirwan, G.M., De Juana, E., 2014. Middle Spotted Woodpecker (Leiopicus medius). En: Del Hoyo, J., Elliott, A., Sargatal, J., Christie, D.A., De Juana, E. (Eds.), Handbook of the Birds of the World Alive. Lynx Edicions, Barcelona. 